\title{
On Two Methods for Approximating Minimal Surfaces in Parametric Form
}

\author{
By Takuya Tsuchiya
}

\begin{abstract}
Two methods for approximating minimal surfaces in parametric form are considered. One minimizes the area of the surface, and the other the energy of the surface. The convergence of the algorithm of the first method is proved. The application of the second method to the approximation of conformal maps is examined. Several examples of computations are given.
\end{abstract}

1. Introduction. There are many papers studying numerical approximations of minimal surfaces but, as far as the author knows, most of them consider minimal surfaces in nonparametric form. In this paper we consider minimal surfaces in parametric form.

Let $\mathbf{R}^{n}(n \geqslant 2)$ be the $n$-dimensional Euclidean space, and let $\mathbf{R}^{2} \supset \Omega$ be a bounded domain with the Lipschitz boundary $\partial \Omega . \mathbf{R}^{n} \supset \Gamma=\left\{\gamma_{1}, \ldots, \gamma_{m}\right\}$ is a system of $m$ Jordan curves, which is homeomorphic to $\partial \Omega$. Let $P C^{\infty}(\Omega)$ be a set of piecewise smooth functions in $\Omega$, and $C(\bar{\Omega})$ be a set of continuous functions on $\bar{\Omega}$, where $\bar{\Omega}$ denotes the closure of the domain $\Omega$. We define the functional space $X$ as follows:

$$
\begin{aligned}
& X \subset\left[P C^{\infty}(\Omega) \cap C(\bar{\Omega})\right]^{n}, \\
& X:=\left\{f: \Omega \rightarrow \mathbf{R}^{n}|f(\partial \Omega)=\Gamma, f|_{\partial \Omega}: \text { monotone }\right\},
\end{aligned}
$$

where monotone means that the inverse image of each point of $\partial \Omega$ is connected. The area functional $A: X \rightarrow \mathbf{R}$ is defined by

$$
A(f):=\iint_{\partial \Omega} \sqrt{\left|f_{x}\right|^{2}\left|f_{y}\right|^{2}-\left(f_{x}, f_{y}\right)^{2}} d x d y
$$

where $f_{x}=\left(f_{1 x}, \ldots, f_{n x}\right), f_{y}=\left(f_{1 y}, \ldots, f_{n y}\right)$ and $|\cdot|,(\cdot, \cdot)$ denote the Euclidean norm and inner product of $\mathbf{R}^{n}$, respectively. Here subscripts $x$ and $y$ mean partial derivatives with respect to $x$ and $y$, respectively.

A stationary point of the area functional $A$ in $X$, even if it is not a minimal point of $A$, is called a minimal surface spanned in $\Gamma$. A minimal surface which is a minimal point of $A$ is called a stable minimal surface. If a minimal surface is not stable, it is called an unstable minimal surface. It is well known that a map is a minimal surface if and only if its mean curvature is zero at any point on the surface

Received August 6, 1984; revised February 7, 1985.

1980 Mathematics Subject Classification. Primary 49D20, 49F10, 65E05.

Key words and phrases. Minimal surface, parametric form, parametrization, conformal map, relaxation method, the Douglas-Rado solution of the classical Plateau problem. 
[8]. In the following section, we give an algorithm for approximating a stationary point of the functional $A$, and we demonstrate the convergence of the algorithm when the functional satisfies several conditions.

From a physical point of view, if the potential energy of a surface is minimal, then the area of the surface will be minimal. In fact, the following holds. Let $\Omega$ be the unit disk, and let $E$ be the energy functional defined by

$$
E(f):=\frac{1}{2} \iint_{\Omega}\left(\left|f_{x}\right|^{2}+\left|f_{y}\right|^{2}\right) d x d y
$$

The minimum value of the energy functional is equal to that of the area functional. And if $f \in X$ attains the minimum value of the energy functional, $f$ is a minimal surface and is (almost) conformal [1], [4].

In Section 3, we consider an approximation of stationary points of the energy functional and conformal maps. In Section 4, we give several numerical examples.

2. The Discrete Area Functional and Its Minimization. Let $\Omega_{h}$ be a regular triangulation of $\Omega$ and $S_{h}$ be a set of maps from $\Omega_{h}$ into $\mathbf{R}^{n}$ which are continuous on $\Omega_{h}$ and are linear on each triangle. Let $\mathbf{b}$ be a suitable parameterization of $\Gamma$ (i.e., $\mathbf{b}$ : $\partial \Omega \rightarrow \Gamma$ is a homeomorphism). Let $X_{h}$ be the subset of $S_{h}$ defined by

$$
X_{h}:=\left\{f_{h} \in S_{h} \mid f_{h}=\mathbf{b} \text { on nodal points of } \partial \Omega_{h}\right\} \text {. }
$$

Note that for any $f \in X$ and any diffeomorphism $\varphi:(\Omega, \partial \Omega) \rightarrow(\Omega, \partial \Omega)$, the area of $f$ is invariant under the action of $\varphi$, that is, $A(f)=A(f \circ \varphi)$. So we may fix the parameterization of $\Gamma$. By the same reason, values of the area functional $A\left(f_{h}\right)$ ( $f_{h} \in X_{h}$ ) depend only on the image of $f_{h}$.

With the usual basis representation for $f_{h} \in S_{h}$, our problem is reduced to the following:

Problem. Find a stationary (minimal) point of a functional

$$
G\left(\mathbf{x}_{1}, \mathbf{x}_{2}, \ldots, \mathbf{x}_{M}\right): \mathbf{R}^{n M} \rightarrow \mathbf{R} \quad \text { with } \quad \mathbf{x}_{i} \in \mathbf{R}^{n} \quad(i=1, \ldots, M),
$$

where $M$ is the number of interior nodal points of $\Omega_{h}$.

A minimal point of $G$ corresponds to a stable minimal surface, and a stationary point which is not a stable point of $G$ corresponds to an unstable minimal surface.

To find stationary (minimal) points of $G$, one may use the usual relaxation method:

$$
\begin{gathered}
G\left(\mathbf{x}_{1}, \ldots, \mathbf{x}_{M}\right)=G\left(a_{1}, \ldots, a_{n M}\right), \quad a_{i} \in \mathbf{R}, i=1, \ldots, n M, \\
a_{i}^{(k+1)}=a_{i}^{(k)}-\omega \frac{G_{a_{i}}\left(a_{i, k}\right)}{G_{a_{i} a_{i}}\left(a_{i, k}\right)},
\end{gathered}
$$

where $a_{i, k}=\left(a_{1}^{(k+1)}, \ldots, a_{i-1}^{(k+1)}, a_{i}^{(k)}, \ldots, a_{n M}^{(k)}\right)$, and $\omega$ is a relaxation parameter. The subscript $a_{i}$ means a partial derivative with respect to $a_{i}$.

However, since the area of the surface is invariant under the action of the diffeomorphism of $\Omega$, the derivative along the "tangent" of the surface is very small 
and " $0 / 0$ " will occur on the right side of (2.2). We therefore define a new relaxation method:

$$
\begin{aligned}
\mathbf{x}_{i}^{(k+1)} & =\mathbf{x}_{i}^{(k)}-\omega \frac{\operatorname{grad}_{i} G\left(\mathbf{x}_{i, k}\right)}{\lambda_{i}\left(\mathbf{x}_{i, k}\right)}, \\
\mathbf{x}_{i, k} & =\left(\mathbf{x}_{1}^{(k+1)}, \ldots, \mathbf{x}_{i-1}^{(k+1)}, \mathbf{x}_{i}^{(k)}, \ldots, \mathbf{x}_{M}^{(k)}\right),
\end{aligned}
$$

where $\operatorname{grad}_{i}=\left(\partial / \partial x_{i 1}, \ldots, \partial / \partial x_{i n}\right), \mathbf{x}_{i}=\left(x_{i 1}, \ldots, x_{i n}\right)$, and $\lambda_{i}\left(\mathbf{x}_{i, k}\right)$ is the maximum eigenvalue of the Hessian

$$
\mathbf{H}_{i}\left(\mathbf{x}_{i, k}\right)=\left(\frac{\partial^{2}}{\partial x_{i h} \partial x_{i l}} G\left(\mathbf{x}_{i, k}\right)\right)_{h, l},
$$

with respect to the $i$ th vector $\mathbf{x}_{i}$.

For the convergence of this algorithm, we have the following proposition. Proposition 1 and its proof are a generalized version of Mittelmann's method in [7].

Suppose that the function $G: \mathbf{R}^{n M} \rightarrow \mathbf{R}$ is bounded from below, twice continuously differentiable and $G(x) \rightarrow \infty$ as $|x| \rightarrow \infty$. For arbitrary $\mathbf{x}_{0} \in \mathbf{R}^{n M}$, we define the set $X_{0}$ by $\mathbf{R}^{n M} \supset X_{0}:=\left\{x \in \mathbf{R}^{n M} \mid G(x) \leqslant G\left(x_{0}\right)\right\}$. Then, by assumption, the set $X_{0}$ is compact.

Proposition 1. Let $\lambda_{i}(\mathbf{x})$ be the maximum eigenvalue of the Hessian $\mathbf{H}_{i}(\mathbf{x})$ with respect to the ith vector $\mathbf{x}_{i}$, defined by (2.4). Assume that there exists a positive constant $\beta>0$ such that $\lambda_{i}(x) \geqslant \beta>0(i=1, \ldots, M)$ for every $x \in X_{0}$.

Then there exists a positive real number $m$ such that if we choose a relaxation parameter $\omega_{i}^{(k)}$ satisfying

$$
0<\omega_{i}^{(k)}<2\left(1+m \frac{\operatorname{grad}_{i} G\left(\mathbf{x}_{i, k}\right) \mid}{\lambda_{i}\left(\mathbf{x}_{i, k}\right)^{2}}\right)^{-1}-\varepsilon,
$$

where $\varepsilon$ is an arbitrary positive real number satisfying

$$
\min _{x \in X_{0}} 2\left(1+m \frac{\operatorname{|grad}_{i} G(\mathbf{x}) \mid}{\lambda_{i}(\mathbf{x})^{2}}\right)^{-1}>\varepsilon>0,
$$

then the sequence defined by (2.3) converges to some $\mathbf{x} \in X_{0}$ and $\mathbf{x}$ is a stationary point of $G$, that is,

$$
\operatorname{grad}_{i} G(\mathbf{x})=0 \quad \text { for } i=1, \ldots, M .
$$

Proof. First, we prove that $G\left(\mathbf{x}_{i+1, k}\right)=G\left(\mathbf{x}_{i, k}\right)$ if and only if $\operatorname{grad}_{i} G\left(\mathbf{x}_{i, k}\right)=0$. It is obvious that $\operatorname{grad}_{i} G\left(\mathbf{x}_{i, k}\right)=0$ implies $G\left(\mathbf{x}_{i+1, k}\right)=G\left(\mathbf{x}_{i, k}\right)$. We prove the inverse statement.

For the function $G(x)$, we use Taylor expansion around $\mathbf{x}_{i, k}$ to obtain

$$
\begin{aligned}
G\left(\mathbf{x}_{i+1, k}\right)= & G\left(\mathbf{x}_{i, k}\right)+\operatorname{grad}_{i} G\left(\mathbf{x}_{i, k}\right) \cdot\left(\mathbf{x}_{i}^{(k+1)}-\mathbf{x}_{i}^{(k)}\right) \\
& +\frac{1}{2}\left(\mathbf{x}_{i}^{(k+1)}-\mathbf{x}_{i}^{(k)}\right)^{T} \mathbf{H}_{i}(\xi)\left(\mathbf{x}_{i}^{(k+1)}-\mathbf{x}_{i}^{(k)}\right),
\end{aligned}
$$

where $\mathbf{H}_{i}(\xi)$ is the Hessian with respect to the $i$ th vector, $\xi=\mathbf{x}_{i, k}+\tau\left(\mathbf{x}_{i+1, k}-\mathbf{x}_{i, k}\right)$, $0 \leqslant \tau \leqslant 1$, and $(\cdot)^{T}$ means transposition. 
For simplicity, we denote $\operatorname{grad}_{i} G\left(\mathbf{x}_{i, k}\right) / \lambda_{i}\left(\mathbf{x}_{i, k}\right), \mathbf{H}_{i}(\xi)$ and $\mathbf{x}_{i}^{(k+1)}-\mathbf{x}_{i}^{(k)}$ by $\mathbf{t}_{i}^{(k)}$, $\mathbf{H}$ and $\mathbf{x}$, respectively.

Since $\mathbf{H}$ is a symmetric matrix, there exists an orthogonal matrix $\mathbf{P}$ such that

$$
\mathbf{H}=\mathbf{P}^{T}\left[\begin{array}{ccc}
\mu_{1} & & 0 \\
& \ddots & \\
0 & & \mu_{n}
\end{array}\right] \mathbf{P},
$$

where $\mu_{j}(j=1, \ldots, n)$ are eigenvalues of the matrix $\mathbf{H}$. From the definition of $\lambda_{i}$, we have

$$
\begin{aligned}
\mathbf{x}^{T} \mathbf{H x} & =(\mathbf{P x})^{T}\left[\begin{array}{lll}
\mu_{1} & & 0 \\
& \ddots & \\
0 & & \mu_{n}
\end{array}\right](\mathbf{P x}) \\
& =\mu_{1}(\mathbf{P x})_{1}^{2}+\cdots+\mu_{n}(\mathbf{P x})_{n}^{2} \leqslant \lambda_{i}|\mathbf{P x}|^{2}=\lambda_{i}|\mathbf{x}|^{2},
\end{aligned}
$$

where $(\mathbf{P x})_{j}$ is the $j$ th component of the vector Px. By means of (2.7), we have

$$
\begin{aligned}
G\left(\mathbf{x}_{i+1, k}\right)-G\left(\mathbf{x}_{i, k}\right) \leqslant & -\omega_{i}^{(k)}\left|\mathbf{t}_{i}^{(k)}\right|^{2} \lambda_{i}\left(\mathbf{x}_{i, k}\right)\left(1-\frac{\omega_{i}^{(k)}}{2}\right) \\
& +\frac{1}{2} \omega_{i}^{(k) 2}\left|\mathbf{t}_{i}^{(k)}\right|^{2}\left(\lambda_{i}(\xi)-\lambda_{i}\left(\mathbf{x}_{i, k}\right)\right) .
\end{aligned}
$$

Since $G$ is of $C^{2}$-class and $X_{0}$ is compact, we can choose a positive constant $m$ satisfying

$$
\begin{aligned}
& \text { if }\left|y-\mathbf{x}_{i}^{(k)}\right| \leqslant 2\left|\mathbf{t}_{i}^{(k)}\right|, \quad y, \mathbf{x}_{i}^{(k)} \in X_{0}, \\
& \text { then }\left|\lambda_{i}(y)-\lambda_{i}\left(\mathbf{x}_{i}^{(k)}\right)\right| \leqslant m\left|\mathbf{t}_{i}^{(k)}\right|, \quad \text { for any } i .
\end{aligned}
$$

Since $\left|\xi-\mathbf{x}_{i, k}\right|=\tau\left|\mathbf{x}_{i+1, k}-\mathbf{x}_{i, k}\right|=\tau \omega_{i}^{(k)}\left|\mathbf{t}_{i}^{(k)}\right| \leqslant 2\left|\mathbf{t}_{i}^{(k)}\right|$, by (2.8), we obtain

$$
G\left(\mathbf{x}_{i+1, k}\right)-G\left(\mathbf{x}_{i, k}\right) \leqslant-\omega_{i}^{(k)} \beta\left|\mathbf{t}_{i}^{(k)}\right|^{2}\left(1-\frac{\omega_{i}^{(k)}}{2}\left(1+\frac{m\left|\mathbf{t}_{i}^{(k)}\right|}{\lambda_{i}\left(\mathbf{x}_{i, k}\right)}\right)\right) \leqslant 0 .
$$
0 .

Then, $G\left(\mathbf{x}^{(k)}\right)$ is decreasing in $k$, and $G\left(\mathbf{x}_{i+1, k}\right)=G\left(\mathbf{x}_{i, k}\right)$ implies $\operatorname{grad}_{i} G\left(\mathbf{x}_{i, k}\right)=$

By assumption, $G$ is bounded from below, so $G\left(\mathbf{x}^{(k)}\right)$ converges to some value. By (2.5), we have

$$
\begin{aligned}
\frac{1}{\beta}\left(G\left(\mathbf{x}_{i, k}\right)-G\left(\mathbf{x}_{i+1, k}\right)\right) & \geqslant\left|\mathbf{x}_{i}^{(k+1)}-\mathbf{x}_{i}^{(k)}\right|^{2}\left(\frac{1}{\omega_{i}^{(k)}}-\frac{1}{2}\left(1+\frac{m\left|\mathbf{t}_{i}^{(k)}\right|}{\lambda_{i}\left(\mathbf{x}_{i, k}\right)}\right)\right) \\
& \geqslant \frac{\varepsilon}{4}\left|\mathbf{x}_{i}^{(k+1)}-\mathbf{x}_{i}^{(k)}\right|^{2} \geqslant 0 .
\end{aligned}
$$

Hence, we obtain the following inequality,

$$
0 \leqslant\left|\mathbf{x}_{i}^{(k+1)}-\mathbf{x}_{i}^{(k)}\right| \leqslant \frac{4}{\varepsilon \beta}\left(G\left(\mathbf{x}_{i, k}\right)-G\left(\mathbf{x}_{i+1, k}\right)\right) .
$$

This implies that if $G\left(\mathbf{x}^{(k)}\right)$ converges, so does $\mathbf{x}^{(k)}$. At the limit point, $G$ is stationary. 
Remark 1. When $G$ is the discrete area functional, it can occur that $G$ does not satisfy the condition of Proposition 1. Then, the relaxation procedure will stop at the step where $\lambda_{i}\left(\mathbf{x}_{i, k}\right)$ in (2.3) is too small. See Example 1.

3. The Classical Plateau Problem and Its Approximation. In this section, we assume that $\Omega \subset \mathbf{R}^{2}$ is a bounded, 1-connected domain with the Lipschitz boundary $\partial \Omega$. So $\Gamma=\left\{\gamma_{1}\right\}$, and $\gamma_{1}$ is homeomorphic to the 1-dimensional unit sphere. In this case, the problem of finding minimal surfaces spanned in $\Gamma$ is called the classical Plateau problem. The classical Plateau problem was solved by Douglas and Rado in 1930 [1], [4]. Here, we formulate the classical Plateau problem as follows:

Taking arbitrary six distinct points $z_{1}, z_{2}, z_{3} \in \partial \Omega$ and $\zeta_{1}, \zeta_{2}, \zeta_{3} \in \Gamma$, we define

$$
X^{\prime}:=\left\{f \in X \mid f\left(z_{i}\right)=\zeta_{i}, i=1,2,3\right\} .
$$

The classical Plateau problem is to find $f \in X^{\prime}$ which is a stationary point of the energy functional $E$ in $X^{\prime}$. It is known that a solution of the classical Plateau problem is a minimal surface spanned in $\Gamma$ and is (almost) conformal, that is, $\left|f_{x}\right|=\left|f_{y}\right|$, and $\left(f_{x}, f_{y}\right)=0$ in $D[1$, pp. 107-118]. In particular, if $f$ is a minimal point of $E$ in $X^{\prime}$, then $f$ is a minimal surface spanned in $\Gamma$. Douglas and Rado proved the following theorem:

Theorem A (Douglas, Rado). If $E_{\Gamma}=\inf \left\{E(g): g \in X^{\prime}\right\}<+\infty$, then there exists $f \in X^{\prime}$ such that $E(f)=E_{\Gamma}$.

Hence there exists at least one minimal surface in $X^{\prime}$ for "good" $\Gamma$. We can show immediately that if $\Gamma$ is rectifiable, then $E_{\Gamma}<+\infty$ [1, pp. $9-10$ and p. 129]. An $f \in X^{\prime}$ satisfying $E(f)=E_{\Gamma}$ is called the classical solution or the Douglas-Rado solution.

Remark 2. When $n=2$, we can prove immediately that the Douglas-Rado solution is conformal [4, p. 71]. Then Theorem A is the Riemann Mapping Theorem for domains bounded by Jordan curves.

The proof of Theorem A tells us that to find a solution of the classical Plateau problem we must find the optimal parameterization of $\Gamma$. Thus, when we consider the minimization of the discrete energy functional, we must move not only interior nodal points in $\mathbf{R}^{n}$ but also boundary nodal points on $\Gamma$, except for three points. We discretize the problem as follows.

First, we determine three fixed points $z_{1}, z_{2}, z_{3}$ on $\partial \Omega$ and corresponding fixed points $\zeta_{1}, \zeta_{2}, \zeta_{3}$ on $\Gamma$. Let $\Omega_{h}$ be a regular triangulation of $\Omega$ such that $z_{1}, z_{2}, z_{3}$ are nodal points of $\Omega_{h}$, and let $N_{h}$ be the set of nodal points. Let $S_{h}$ be a set of maps from $\Omega_{h}$ into $\mathbf{R}^{n}$ which are continuous on $\Omega_{h}$ and are linear on each triangle. We define functional spaces $X_{h}, X_{h}^{\prime}$ by

$$
\begin{gathered}
X_{h}:=\left\{f_{h} \in S_{h}\left|f_{h}\left(\Omega_{h} \cap N_{h}\right) \subset \Gamma, f_{h}\right|_{\Omega_{h} \cap N_{h}}: d \text {-monotone }\right\}, \\
X_{h}^{\prime}:=\left\{f_{h} \in X_{h} \mid f\left(z_{i}\right)=\zeta_{i}, i=1,2,3\right\}
\end{gathered}
$$

where $d$-monotone means that the order of nodal points $f_{h}\left(\Omega_{h} \cap N_{h}\right)$ on $\Gamma$ is the same as the order of nodal points on the boundary of $\Omega_{h}$. 
With the usual basis representation for $f \in S_{h}$ and a parameterization of $\Gamma$, the energy functional $E$ is reduced to a functional $H\left(x_{1}, \ldots, x_{n M}, x_{n M+1}, \ldots, x_{n M+N}\right)$ : $\mathbf{R}^{n M+N} \rightarrow \mathbf{R}$ with $-\infty<x_{i}<+\infty$ for $i=1, \ldots, n M$ and $w_{1} \leqslant x_{n M+1} \leqslant \cdots \leqslant w_{2}$ $\leqslant \cdots \leqslant w_{3} \leqslant \cdots \leqslant x_{n M+N} \leqslant w_{1}+2 \pi$, where $w_{1}, w_{2}, w_{3}$ are constants corresponding to the given fixed points $\zeta_{1}, \zeta_{2}, \zeta_{3}$. We look for stationary points $f_{h} \in X_{h}^{\prime}$ of $H$, that is, grad $H\left(f_{h}\right)=0$.

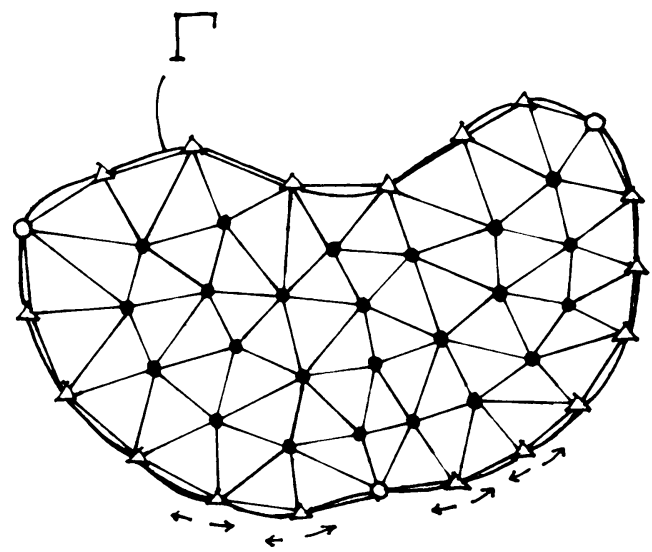

FIGURE 1

The finite element scheme for the energy functional.

" $O$ " is a fixed nodal point.

" $\Delta$ " is a boundary nodal point moving on $\Gamma$.

"๑" is an interior nodal point moving $n$-dimensionally.

As relaxation procedure we may use the usual one, (2.2).

4. Numerical Examples. In this section, we give several numerical examples.

Example 1. Catenoid. Let $f(x)=\cosh (a(x-c)) / a, 0 \leqslant x \leqslant 1$. The constants $a$ and $c$ are determined by the boundary conditions. Rotating the graph of $y=f(x)$ around the $x$-axis, we get a catenoid, which is known as an example of a minimal surface. Now we set $f(0)=f(1)=r$, where $r$ is a given constant. Then $c=0.5$, and the constant $a$ is determined by the equation $\cosh (a / 2)=a r$. If $r$ is smaller than a certain constant, this equation has no real solutions. The critical value is $r_{0}=0.75444$.

The initial data for the relaxation are cylindrical ones. We denote by $\alpha$ the right side of (2.5). The relaxation parameter $\omega$ is 1.2 when $\alpha \geqslant 1.3$ and $\alpha \times 0.923$ when $\alpha<1.3$.

In Table 1, we give iteration numbers, distances between a numerical solution and the $x$-axis and its exact value for a given $r$. The iterations were terminated when $\max \left|x_{i}^{(k)}-x_{i}^{(k-1)}\right|<10^{-5}$. As $r$ approaches the critical value $r_{0}$, the iteration number is increasing. We give the numerical solution for $r=0.756$ in Figure 2 .

When $r \leqslant 0.755$, the minimizing surface collapses, and, at some step of the iteration, the relaxation procedure cannot continue, since $\lambda_{i}\left(\mathbf{x}_{i, k}\right)$ in (2.3) becomes very small. The iteration was terminated when $\lambda_{i}\left(\mathbf{x}_{i, k}\right)<10^{-20}$. In Table 2 , we give numbers of iterations at which termination occurred. 
TABLE 1

Results of computations for Catenoid

\begin{tabular}{|l|c|c|c|c|}
\hline \multicolumn{1}{|c|}{$r$} & count & $x$ & distance & exact value \\
\hline 1.0 & 234 & 0.50142 & 0.84762 & 0.84834 \\
0.9 & 272 & 0.50178 & 0.71807 & 0.71904 \\
0.8 & 395 & 0.50234 & 0.56122 & 0.56299 \\
0.79 & 429 & 0.50241 & 0.54117 & 0.54314 \\
0.78 & 479 & 0.50250 & 0.51894 & 0.52121 \\
0.77 & 560 & 0.50259 & 0.49299 & 0.49574 \\
0.76 & 790 & 0.50268 & 0.45757 & 0.46190 \\
0.757 & 1037 & 0.50267 & 0.44036 & 0.44675 \\
0.756 & 1272 & 0.50264 & 0.43126 & 0.43994 \\
\hline
\end{tabular}

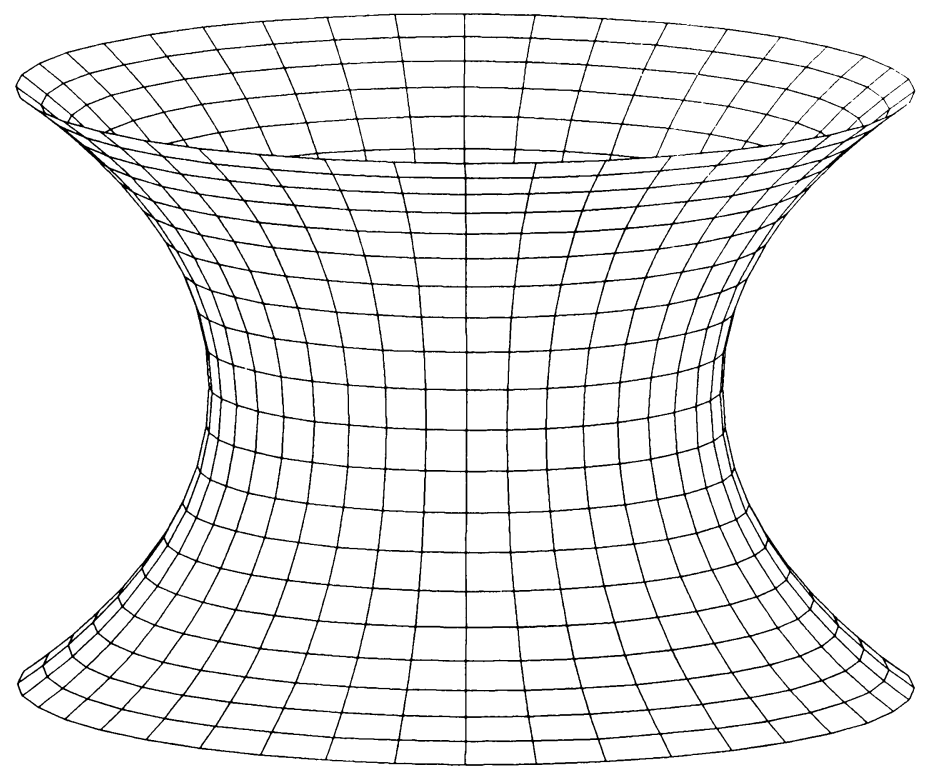

FIGURE 2

Catenoid, $r=0.756$.

TABLE 2

Iteration numbers

\begin{tabular}{|l|r|}
\hline \multicolumn{1}{|c|}{$r$} & count \\
\hline 0.7 & 462 \\
0.74 & 899 \\
0.75 & 1340 \\
0.752 & 1586 \\
0.753 & 1840 \\
0.754 & 2302 \\
0.755 & 3448 \\
\hline
\end{tabular}


As $r$ approaches the critical value, the speed of "convergence" decreases. Figure 3 is the collapsed surface for $r=0.755$.

Example 2. Courant's Example. We take a contour like Figure 4. This is an interesting example, because there are two different minimal surfaces in this contour $[1$, p. 120]. This example was examined in [3].

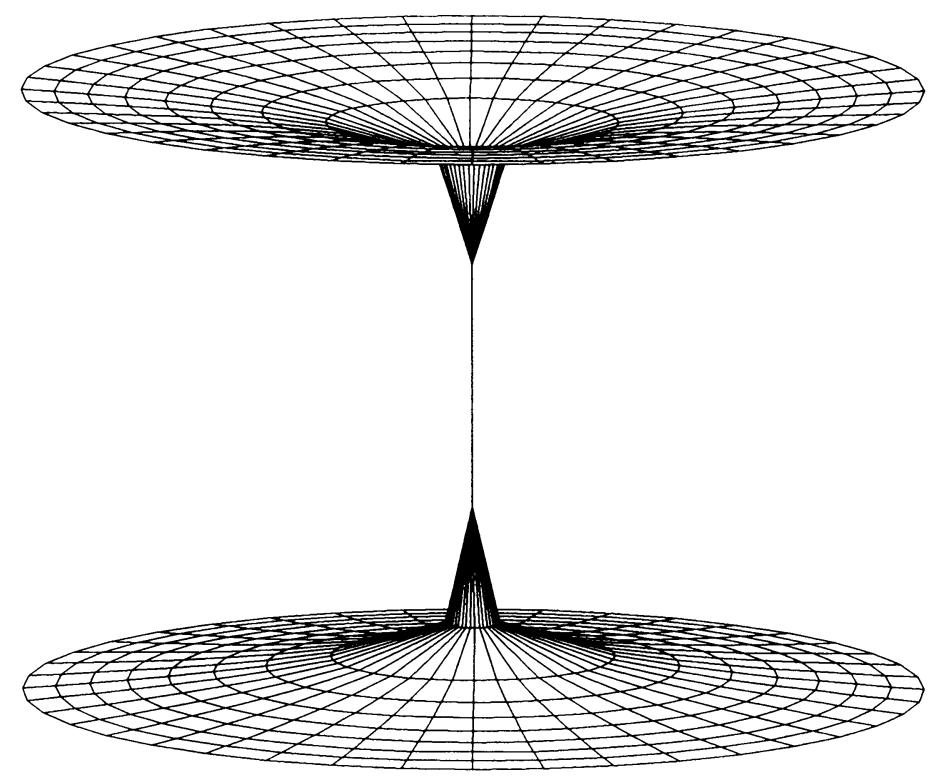

Figure 3

Collapsed catenoid, $r=0.755$.

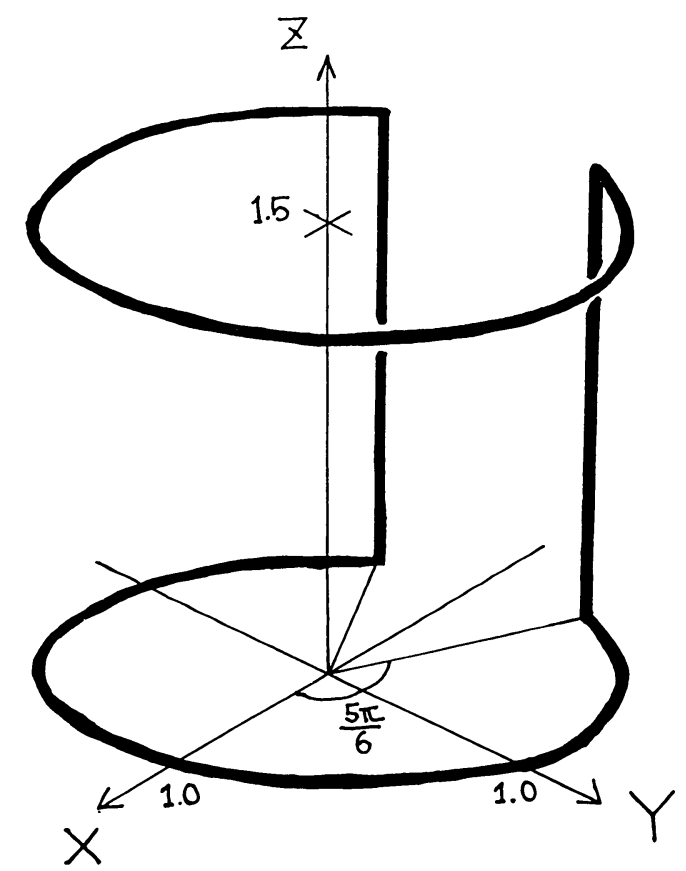

Figure 4

The contour for Example 2. 
Figures 5 and 6 are the results of computations.

Example 3. Minimal Surfaces in a Knot. We approximate minimal surfaces spanned in a knot. The contour is $x=(1+0.25 \cos 3 \theta) \cos 2 \theta, \quad y=$ $(1+0.25 \cos 3 \theta) \sin 2 \theta, z=0.25 \sin 3 \theta, 0 \leqslant \theta \leqslant 2 \pi$. We compute a stationary point of the energy functional. Figure 7 is the result. The area is 6.7424374 and the energy is 6.7685022 .

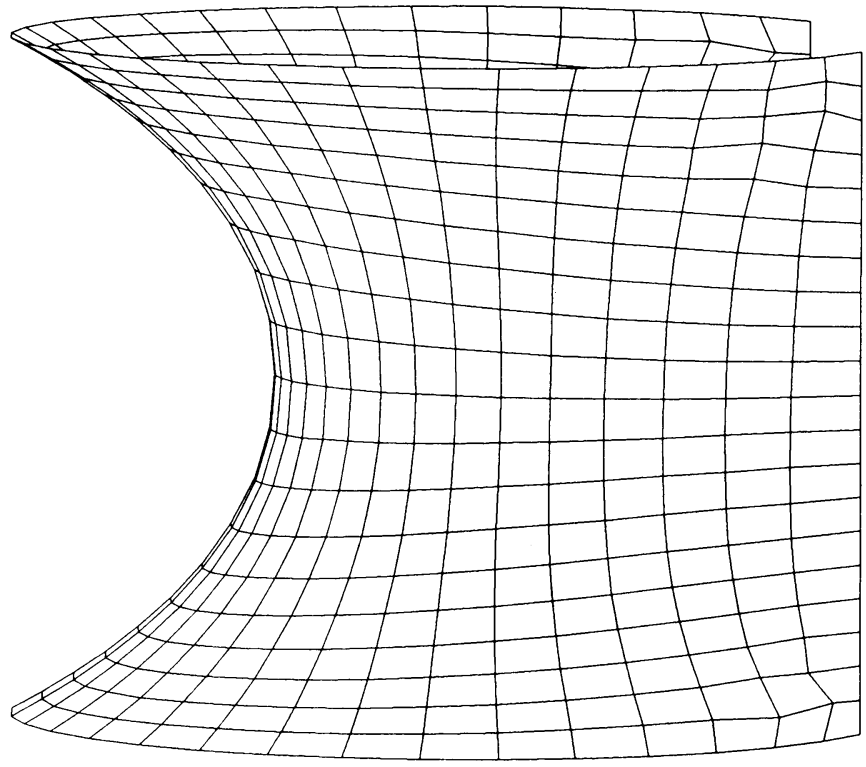

Figure 5

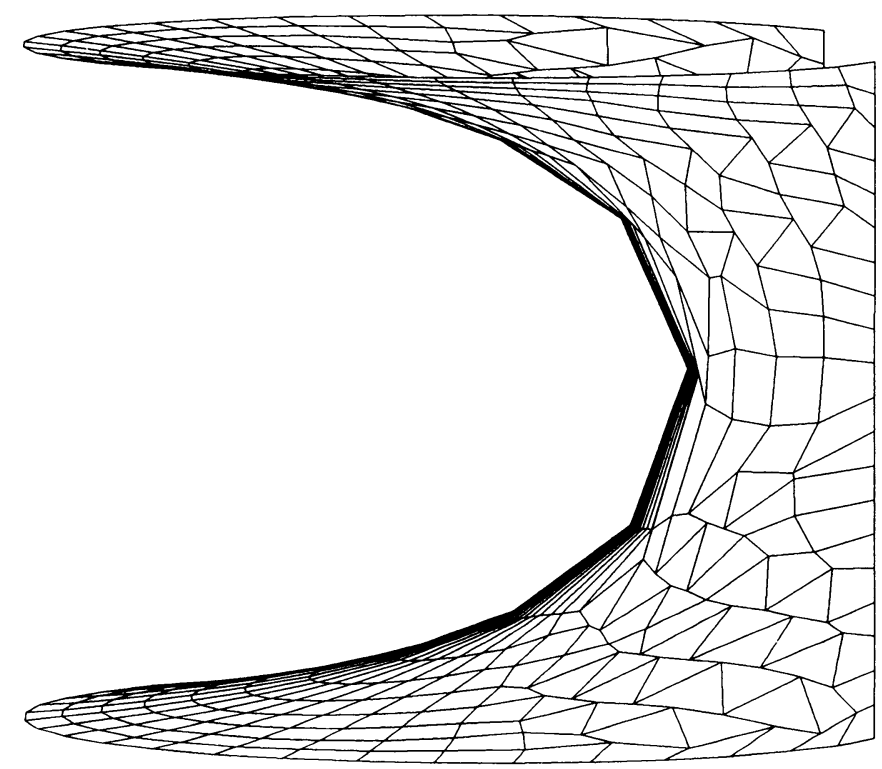

FIGURE 6 
This surface is not necessarily a minimal point of $E$. It is known that, if there exist two stable minimal surfaces spanned in $\Gamma$, then there exists an unstable minimal surface spanned in $\Gamma$ [1, pp. 223-243].

This surface has a branch point. Thus, this is not the Douglas-Rado solution, because the Douglas-Rado solution does not have any branch point when $n=3[4$, p. 80].

We remark that the relaxation procedure of the discrete area functional using the algorithm (2.3) with this surface as initial data is terminated in several iterations.

Figure 8 is another result with a contour $x=(1+0.15 \cos \theta) \cos 2 \theta, y=$ $(1+0.15 \cos \theta) \sin 2 \theta, z=0.15 \sin \theta, 0 \leqslant \theta \leqslant 2 \pi$. The area is 6.3725386 and the energy is 6.3873806 .

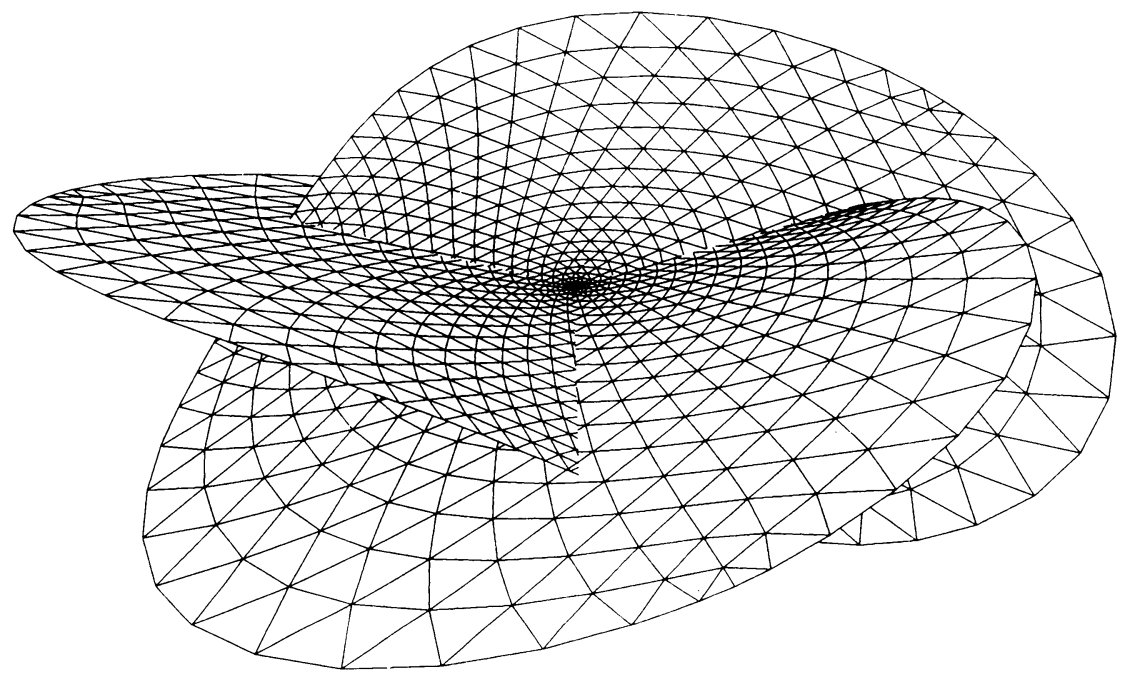

FIGURE 7

The surface which attains the minimal value of the energy functional. The contour is a "clover knot". The energy is 6.7685022. The area is 6.7424374 .

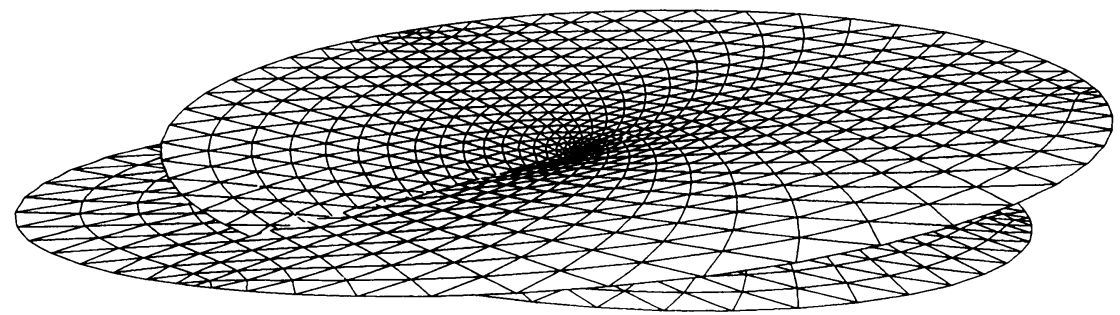

FIGURE 8

Another result of Example 3. The contour is a twisted knot. The energy is 6.3873806. The area is 6.3725386 . 
Example 4. Conformal Maps. Let $n=2$. We approximate conformal maps from a hexagon into bounded, 1-connected domains. The contours are given by $x=$ $(1+R \cos 3 \theta) \cos \theta, y=(1+R \cos 3 \theta) \sin \theta, 0 \leqslant \theta \leqslant 2 \pi$. The minimum value of the energy is the area of the domain surrounded by the contour, and in this case, the value is $\pi\left(1+R^{2} / 2\right)$. Figure 9 is a triangulation of the hexagon, the Figures 10 and 11 are the results for $R=0$ and $R=0.5$, respectively.

All computations were carried out using the FACOM M382 computer system at the Computer Center, Kyushu University.

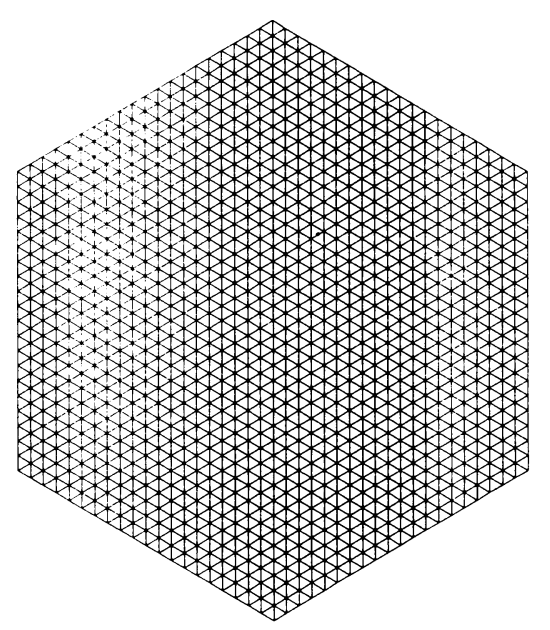

FIGURE 9

Triangulation of hexagon.

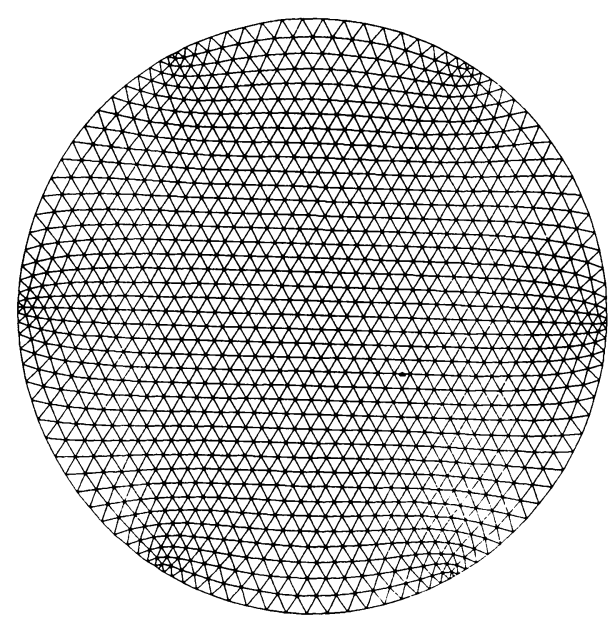

FIGURE 10

$R=0.0$. The energy is 3.1407843 .

The exact value of the energy is 3.1415926 . 


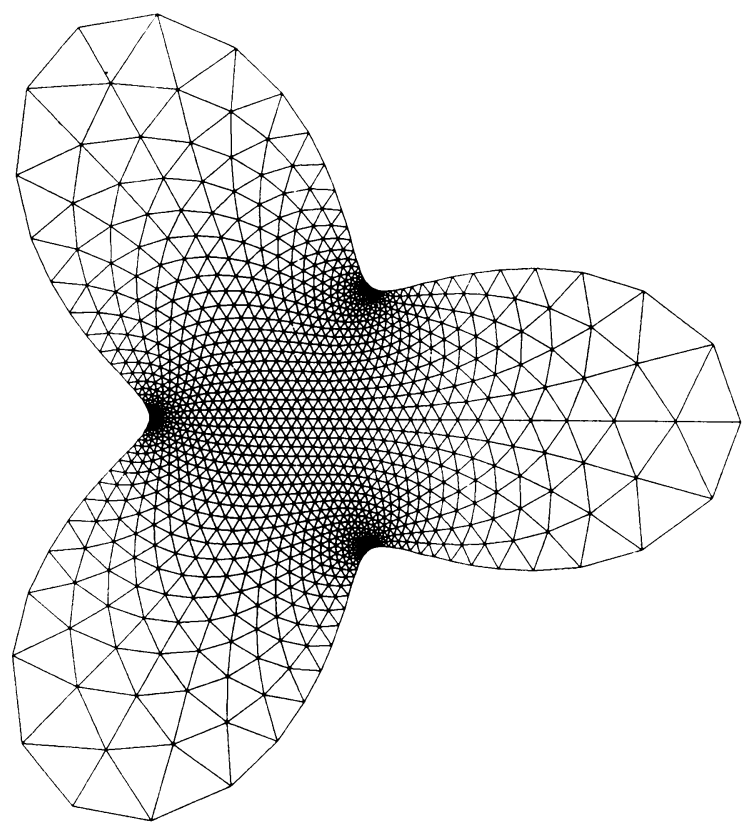

FIGURE 11

$R=0.5$. The energy is 3.5237980 .

The exact value of the energy is 3.5342917.

5. Conclusions. We examined two methods for approximating minimal surfaces in parametric form. Numerical examples presented here show that these methods have sufficient accuracy from a practical point of view.

In this paper, we have not given the error analysis of these methods. Since the area of a surface is invariant under the action of a diffeomorphism of $\Omega$, the error analysis of the discrete area functional will be very difficult. Some results about the convergence of the discrete solutions of the classical Plateau problem will be given elsewhere by the author.

Acknowledgment. The 3-dimensional graphics in this paper are due to Professor Yoshio Ohno of the Institute of Information Science, Keio University. The author is very grateful to him for making his programs available.

Department of Mathematics

Kyushu University 33

Fukuoka, 812, Japan

1. R. Courant, Dirichlet's Principle, Conformal Mapping, and Minimal Surfaces, Interscience, New York, 1950.

2. R. Courant \& D. Hilbert, Methods of Mathematical Physics, vol. II, Interscience, New York, 1962.

3. M. Hinata, M. Shimasaki \& T. KiYono, "Numerical solution of Plateau's problem by a finite element method," Math. Comp., v. 28, 1974, pp. 45-60. 
4. H. B. Lawson, Lectures on Minimal Submanifolds, Vol. I, Publish or Perish, Berkeley, 1980.

5. H. D. MittelmanN, "On the approximation of capillary surface in a gravitational field," Computing, v. 18,1977 , pp. 141-148.

6. H. D. MittelmanN, "On the pointwise estimates for a finite element solution of nonlinear boundary value problems," SIAM J. Numer. Anal., v. 14, 1977, pp. 773-778.

7. H. D. Mittelmann, "On the approximate solution of nonlinear variational inequalities," Numer. Math., v. 29, 1978, pp. 451-462.

8. R. Osserman, A Survey of Minimal Surfaces, Van Nostrand Reinhold, New York, 1969. 